\title{
Probing QCD approach to thermal equilibrium with ultrahigh energy cosmic rays
}

\author{
Jorge F. Soriano* \\ Department of Physics \& Astronomy, Lehman College, CUNY, NY 10468, USA \\ Department of Physics, Graduate Center, City University of New York, NY 10016, USA \\ E-mail: jfdezsoriano@gmail.com
}

\section{Luis A. Anchordoqui}

Department of Physics \& Astronomy, Lehman College, CUNY, NY 10468, USA Department of Physics, Graduate Center, City University of New York, NY 10016, USA

Department of Astrophysics, American Museum of Natural History, NY 10024, USA

E-mail: luis.anchordoqui@gmail.com

\section{Thomas C. Paul}

Department of Physics \& Astronomy, Lehman College, CUNY, NY 10468, USA

E-mail:tompaulsteregmail.com

\section{Thomas J. Weiler}

Department of Physics \& Astronomy, Vanderbilt University, Nashville, TN 37235, USA

E-mail: tom.weiler@vanderbilt.edu

The Pierre Auger Collaboration has reported an excess in the number of muons of a few tens of percent over expectations computed using extrapolation of hadronic interaction models tuned to accommodate LHC data. Very recently, we proposed an explanation for the muon excess assuming the formation of a deconfined quark matter (fireball) state in central collisions of ultrarelativistic cosmic rays with air nuclei. At the first stage of its evolution the fireball contains gluons as well as $u$ and $d$ quarks. The very high baryochemical potential inhibits gluons from fragmenting into $u \bar{u}$ and $d \bar{d}$, and so they fragment predominantly into $s \bar{s}$ pairs. In the hadronization which follows this leads to the strong suppression of pions and hence photons, but allows heavy hadrons to be emitted carrying away strangeness. In this manner, the extreme imbalance of hadron to photon content provides a way to enhance the muon content of the air shower. In this communication we study theoretical systematics from hadronic interaction models used to describe the cascades of secondary particles produced in the fireball explosion. We study the predictions of one of the leading LHC-tuned models QGSJet-II-04 considered in the Auger analysis.

35th International Cosmic Ray Conference - ICRC2017

10-20 July, 2017

Bexco, Busan, Korea

${ }^{*}$ Speaker. 
Besides addressing central questions in ultrahigh energy cosmic ray (UHECR) astrophysics - determining the baryonic component and identifying or constraining sources - the AugerPrime upgrade will provide unique access to particle physics at an order-of-magnitude higher center-ofmass energy than the Large Hadron Collider (LHC) [1].

As recently demonstrated by the Pierre Auger Collaboration [2], it is possible to test particle physics well above $100 \mathrm{TeV}$ in the UHECR-air nucleon center-of-mass energy, using hybrid UHECR air showers, even with a mixed primary composition. Moreover, the column energydensity in UHECR-air collisions is an order of magnitude greater than in $\mathrm{Pb}-\mathrm{Pb}$ collisions at the LHC, suggesting the potential for new hadronic physics from gluon saturation and possibility of exploring quark-gluon plasma as well as quark matter formation by heavy nuclear primaries at far higher energies than available in accelerators $[3,4]$.

A significant discrepancy in the shower muon content is found (greater than $2 \sigma$, statistical and systematics combined in quadrature) between predictions of LHC-tuned hadronic event generators and observations [2]. With the added muon-electromagnetic separation and the significantly higher data-taking rate for the highest energy hybrid events provided by AugerPrime, the reason for this discrepancy may be determined.

As a UHECR-induced air shower develops in the atmosphere, it increases in particle number, before eventually the particle energies drop below some threshold, at which ionization losses begin to cull the particle population. The position at which an air shower deposits the maximum energy per unit mass traversed is known as $X_{\max }$ and its dispersion is known as $\sigma\left(X_{\max }\right)$. Both of these observables are sensitive to the primary UHECR composition, though interpreting this dependence requires resorting to predictions of simulations of hadronic interactions at energies and in kinematical regions inaccessible to terrestrial experiments [5].

The Auger surface array can also be employed to extract composition-dependent information, including the shower muon richness observed a the ground as well as the muon production depth, $X_{\text {nax }}^{\mu}$, which is the depth along the shower axis where muon production reaches a maximum. One advantage of the surface array is its nearly $100 \%$ duty cycle, compared to the roughly $15 \%$ duty cycle of the fluorescence detectors. A measure of $X_{\max }^{\mu}$ can be attained via timing information of the muons when they arrive at the ground. Curiously, the primary masses predicted by $X_{\max }$ and $X_{\max }^{\mu}$ are not in agreement, with the $X_{\max }$ suggesting an average composition at the highest energies in the mass range of nitrogen [6], while $X_{\max }^{\mu}$ is more consistent with a composition heavier than iron, using LHC-tuned EPOS at the hadronic interaction model [7]. Using QGSJET leads to a better agreement between $X_{\max }$ and $X_{\max }^{\mu}$, with $X_{\max }^{\mu}$ favoring iron composition at the highest energies. Regardless of models chosen, however, it appears difficult at present to reconcile the $X_{\max }$ and $X_{\max }^{\mu}$ measurements with each other using existing hadronic interaction models.

In addition, it is interesting to note that while for $10^{9.5} \lesssim E / \mathrm{GeV} \lesssim 10^{10.6}$ the mean and dispersion of $X_{\max }$ inferred from fluorescence Auger data point to a light composition (protons and helium) towards the low end of this energy bin and to a large light-nuclei content (around helium) towards the high end (see Fig. 3 in [8]), when the signal in the water Cherenkov stations (with sensitivity to both the electromagnetic and muonic components) is correlated with the fluorescence data, a light composition made up of only proton and helium becomes inconsistent with observations [9]. The hybrid data indicate that intermediate nuclei, with baryon number $A=14$, must contribute to the energy spectrum in this energy bin. Moreover, a potential iron contribution cannot 
be discarded.

New physics processes in the first interaction would tend to increase $\sigma\left(X_{\max }\right)$, making the nuclear composition appear lighter than what actually is. This is because we would not expect new physics processes when nuclei just slide along each other. The admixture of peripheral and "new physics" collisions would then produce large fluctuations in the number of muons at ground level and increase $\sigma\left(X_{\max }\right)$. On the other hand, $X_{\max }^{\mu}$ occurs after the shower is more fully thermalized and hence $\sigma\left(X_{\max }^{\mu}\right)$ is less sensitive to new physics in the first interaction. Combining information from these two variables thus provides a means of disentangling effects of nuclear composition from new physics. If measurements of $\sigma\left(X_{\max }\right)$ and $X_{\max }^{\mu}$ were equally accessible expermentally, $X_{\max }^{\mu}$ would provide a better measurement of the nuclear composition, as it is less sensitive to new physics in the first interaction.

It is also important to note that any new physics should affect $\sigma\left(X_{\text {nax }}\right)$, causing the distribution to trend upwards above the new physics threshold, an effect which would be too large to be accounted for by increasing primary mass. On the other hand, new physics would not manifest in this way in the case of $X_{\max }$. A constant nuclear composition would yield a straight elongation rate, while an increase in primary mass would cause $X_{\max }$ to inflect downward. These unique predictions for $X_{\max }$ and $\sigma\left(X_{\max }\right)$, are in agreement with existing data; see again Fig. 3 in [8]. With enough statistics, it should be possible to discern these effects.

Very recently we presented a model that can accommodate all these anomalies [4]. The model builds up on an old idea, which allows formation of a deconfined quark matter (fireball) state in central collisions of ultrarelativistic cosmic rays with air nuclei [10]. At the first stage of its evolution the fireball contains gluons as well as $u$ and $d$ quarks. The very high baryochemical potential inhibits gluons from fragmenting into $u \bar{u}$ and $d \bar{d}$, and so they fragment predominantly into $s \bar{s}$ pairs. In the hadronization which follows this leads to the strong suppression of pions and hence photons, but allows heavy hadrons to be emitted carrying away strangeness. In this manner, the extreme imbalance of hadron to photon content provides a way to enhance the muon content of the air shower. In addition, the admixture of peripheral and fireball collisions would then produce large fluctuations on $X_{\max }$, but the muon shower maximum $X_{\max }^{\mu}$ would have small fluctuations, in agreement with existing data.

In this paper we use simulations to study the effects of fireball models in air shower observables. Cosmic Ray Monte Carlo (CRMC v1.6.0) [12] is an interface to different hadronic event generators such as EPOS LHC [13] or QGSJETII-04 [14]. It outputs the secondaries of particle interactions for different hadronic models, which allows an standard use of the interaction products for any further analysis. CORSIKA (v7.5600) [15] performs the simulation of atmospheric showers produced after cosmic ray interactions in the atmosphere. It makes use of different hadronic event generators to obtain the products of the first and subsequent interactions at the highest energies. Nevertheless, the first interaction can be treated externally (STACKIN option), allowing the user to give as input not just the cosmic ray primary, but the secondaries and their energies and momenta. It is possible then to modify the particle content and properties after the first interaction to analyze afterwards the evolution of the shower.

Our goal is to test whether fireball-like models would be able to mitigate the current tension between LHC extrapolated predictions and data at the Pierre Auger Observatory, without developing a full theoretical framework for fireball models or carrying out fully detailed simulations of the 

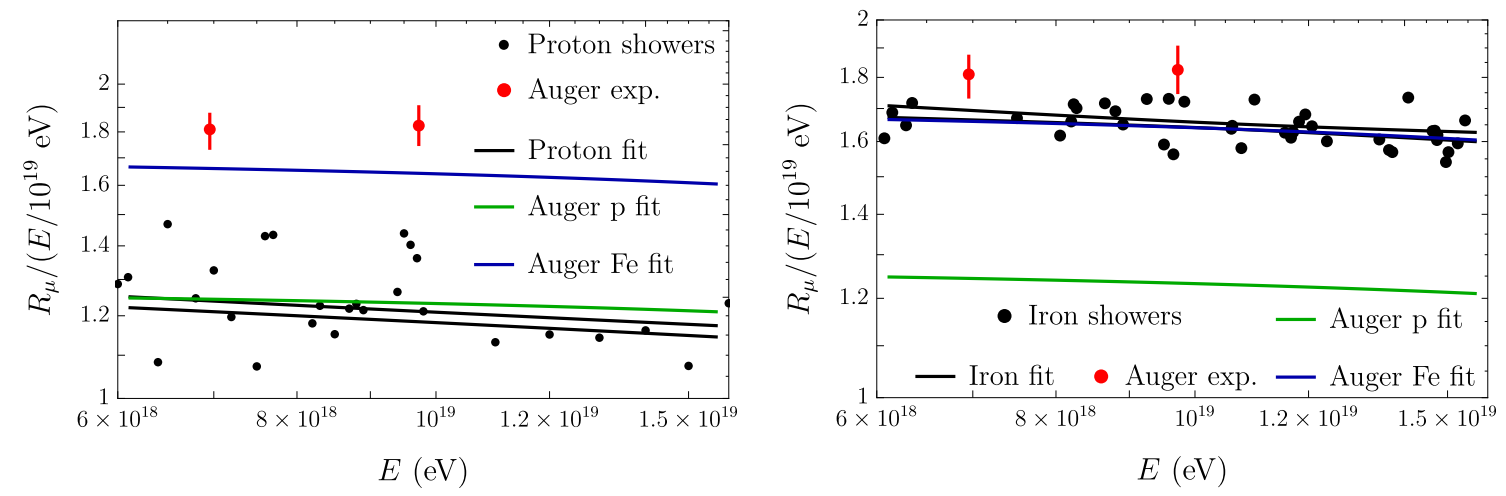

Figure 1: Simulated showers (proton at left, iron at right) and $68 \%$ C. L. fit bands, Auger fits to proton and iron simulations, and Auger measures. All simulations using QGSJET II-04.

physical processes involved in fireball interaction secondary particle production. In this work we take CRMC results for first interactions and modify the particle content in a consistent manner according to the above description of the fireball model, where the pion content is highly suppressed. We study the effect of this modification on the muon content at the Pierre Auger observation level.

The computational power needed to run full simulations of atmospheric showers is an important limiting factor in the accuracy that one can reach in simulated air shower analyses. The thinning option in CORSIKA allows a reduction of the computing time by reducing some information about the development of individual particles and compensating the by applying weights to simulated particles. A comparison of results for thinned and unthinned showers presented in [16] allows us to consider that, when the expected number of particles at observation level is high enough, the fluctuations (of the number of particles) created by the thinning algorithm are small enough for the result to be close to the corresponding unthinned result. Nevertheless, due to time constraints, we use a less conservative thinning threshold than the one presented in [16], which may be source of some uncertainties in our results.

In order to measure the reliability of our simulations, we perform an analysis of the muon content at ground level with the parameters suggested in [11] for comparison. We concentrate on the energy interval $10^{9.9} \lesssim E / \mathrm{GeV} \lesssim 10^{10.2}$, and simulate proton showers at $67^{\circ}$ zenith angle. Following [11] a fit to a parametrization

$$
\left\langle R_{\mu}\right\rangle=a\left(E / 10^{19} \mathrm{eV}\right)^{b}
$$

is assumed. Here, $R_{\mu}=N_{\mu} / N_{\mu, 19}$, where $N_{\mu}$ the number of muons at the Auger observation level above $0.3 \mathrm{GeV}$ for any energy, and $N_{\mu, 19}$ the same quantity evaluated at $10^{19} \mathrm{eV}$. Fitting (1) to our data we obtain $a=1.24 \pm 0.05$ and $b=0.90 \pm 0.02$, in good agreement with the values presented in [11]. This agreement can be well appreciated in Fig. 1.

The next step is to perform the same kind of analysis using primary particles that may create fireballs in the first interaction. We take iron $(\mathrm{A}=56)$ as reference. A new fit of (1) to data gives $a=1.65 \pm 0.01$ and $b=0.94 \pm 0.02$. The data and fit can be also seen in Fig. 1 . 

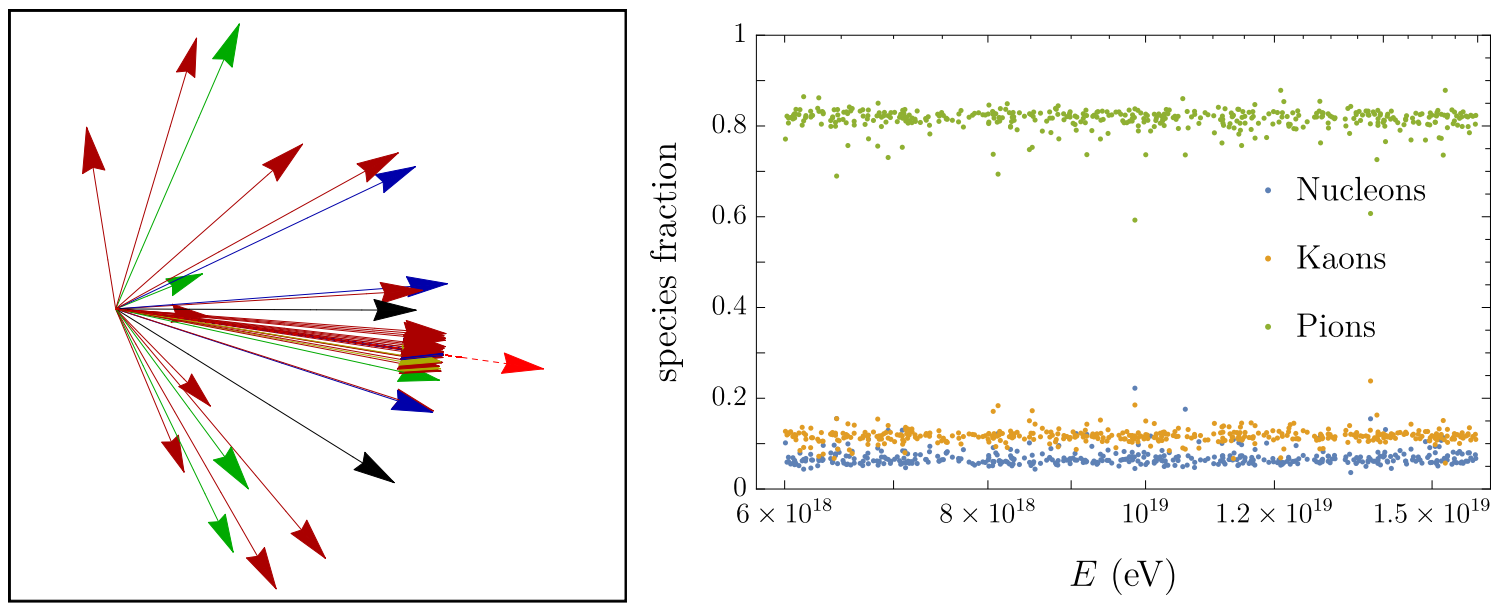

Figure 2: Left: Secondaries normalized momenta in a N-N interaction in the target rest frame. Dashed red arrow points to shower axis. Colors represent particle species (green $=$ nucleons, red $=$ pions, blue $=$ kaons, yellow=photons, black = others). The transverse Right: fraction of kaons, pions and nucleons after the first interaction.

A Heitler model predicts the total number of muons in a shower to follow

$$
N_{\mu, \mathrm{tot}}=A\left(\frac{E / A}{E_{c}}\right)^{\alpha}
$$

where $E$ and $A$ are the primary energy and mass number, respectively, $E_{c}$ the critical energy for $\pi^{ \pm}$ to decay into $\mu^{ \pm}$. If we assume $R_{\mu} \propto N_{\mu \text {,tot }}$, the exponents $\alpha$ and $b$ in (2) and (1) must be the same. Since $b$ is almost the same for proton and iron primaries, $\left\langle R_{\mu}\right\rangle_{\mathrm{Fe}} /\left\langle R_{\mu}\right\rangle_{\mathrm{p}} \sim 56^{1-b} \approx 0.89 \pm 0.07$, where we have used the value of $b$ from [11]. Using our fit parameters we obtain

$$
\left\langle\frac{\left\langle R_{\mu}\right\rangle_{\mathrm{Fe}}}{\left\langle R_{\mu}\right\rangle_{\mathrm{p}}}\right\rangle \approx 1.13 \pm 0.05,
$$

where the external average is taken to be the considered energy interval. Due to the approximate nature of the previous estimations, the numbers can be considered to be in agreement, and this serves as another validation of the overall good behavior of our analysis with the selected thinning.

Now, we proceed to the simulation and analysis of individual first interactions. Using CRMC to interface with QGSJET II-04 we produce iron-nitrogen collisions in the energy interval of our interest. We extract the different particles created in the interaction, together with their energies, momenta and child particles (in the case of the prompt decay of neutral pions and kaons). Since we are precisely interested in those neutral mesons, we revert those decays before performing any analysis. An example of the secondary particles created in those interactions is shown in Fig. 2.

On the right panel of Fig. 2 we extract the information about the fraction of particles of each kind, considering nucleons, pions and kaons. As expected, the number of pions outnumbers that of kaons. As a first approximation to see if the fireball model produces effects in the adequate direction to explain current data, we invert kaon and pion populations. According to Fig. 2, the proportion of kaons will be around $80 \%$, much higher than the one of pions. This is, as stated previously, a first approach to the effect that one would expect from a fireball model. 

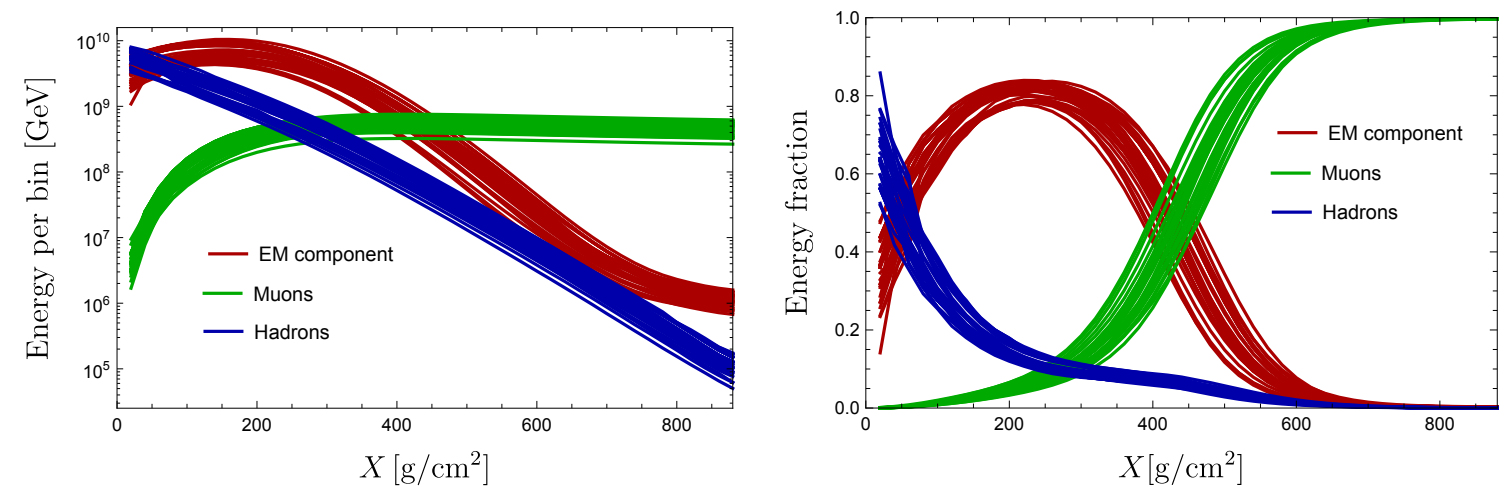

Figure 3: Energy distribution and fraction of total energy for proton showers.
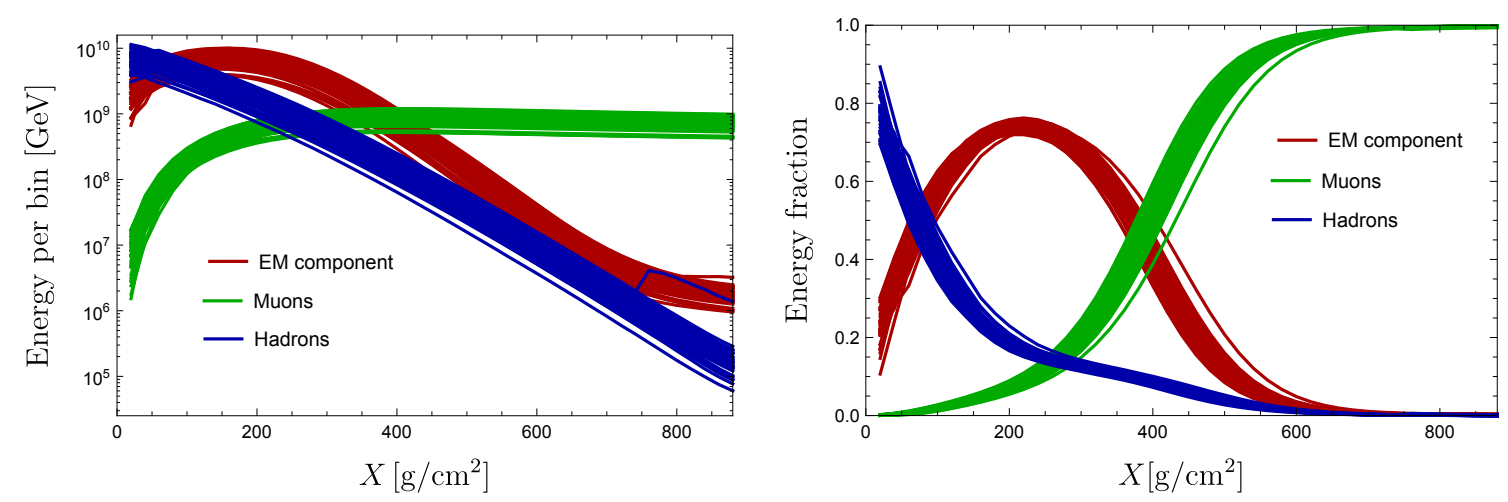

Figure 4: Energy distribution and fraction of total energy for iron showers.
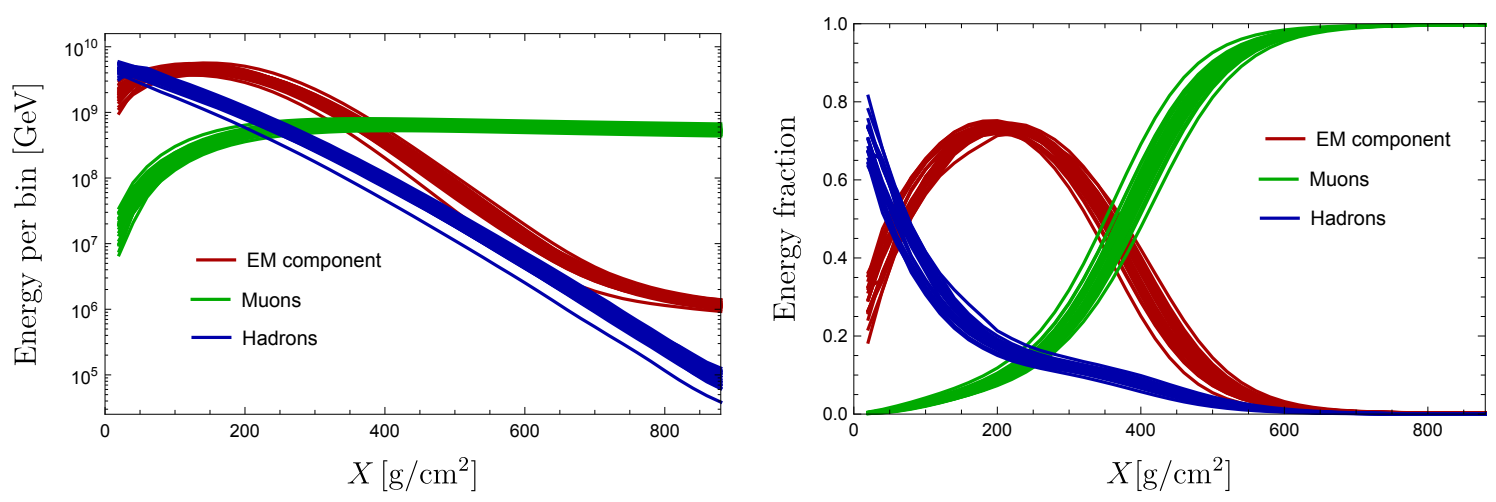

Figure 5: Energy distribution and fraction of total energy for modified iron showers.

In order to compare the evolution of the shower in the different situations, we show (Figs. 3,4 and 5) the energy distribution and fraction of total energy for the electromagnetic, muonic and hadronic components of the different showers. One can see how the energy in the muonic component increases for iron showers, as commented before. Note that the range of energies for the showers in Fig. 5 is much smaller than the used in 3 and 4.

In order to see more precisely how increasing the amount of energy in the kaon sector after 


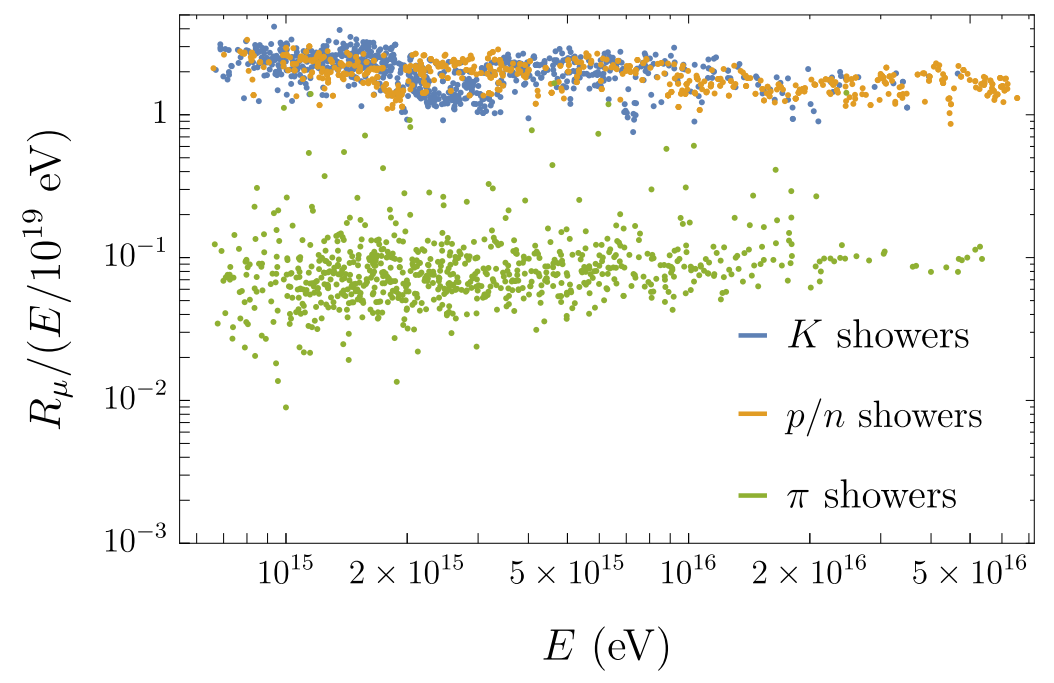

Figure 6: Energy distribution and fraction of total energy for modified iron showers.

the first interaction would increase the number of muons at ground, one can study kaon, pion and proton showers in the typical energy range of the products coming from primaries in the studied energy range. In Fig. 6 we show the number of muons at ground for those kind of showers. While proton/neutron showers provide a similar amount of muons than kaon showers, pion showers remain around one order of magnitude lower. Then, transferring the energy contained in pions to kaons would produce the expected effect of increasing $R_{\mu}$.

A precise determination of the increase in $R_{\mu}$ that would follow from a given modification of the particle content after the first interaction is still ongoing and will be presented elsewhre.

\section{Acknowledgments}

We would like to acknowledge many useful discussions with our colleagues of the Pierre Auger Collaboration. This work has been partially supported by the U.S. National Science Foundation (NSF) Grant No. PHY-1620661 (JFS and LAA), by the National Aeronautics and Space Administration (NASA) Grant No. NNX13AH52G (LAA and TCP), and by the U. S. Department of Energy (DoE) Grant No. DESC-0011981 (TJW).

\section{References}

[1] A. Aab et al. [Pierre Auger Collaboration], arXiv:1604.03637.

[2] A. Aab et al. [Pierre Auger Collaboration], Phys. Rev. Lett. 117, no. 19, 192001 (2016).

[3] G. R. Farrar and J. D. Allen, EPJ Web Conf. 53, 07007 (2013).

[4] L. A. Anchordoqui, H. Goldberg and T. J. Weiler, Phys. Rev. D 95, 063005 (2017).

[5] L. Anchordoqui et al., Annals Phys. 314, 145 (2004).

[6] A. Aab et al. [Pierre Auger Collaboration], Phys. Rev. D 90, 122005 (2014); 90, 122006 (2014). 
[7] A. Aab et al. [Pierre Auger Collaboration], Phys. Rev. D 90012012 (2014); 90, 039904 (2014); 92, 019903 (2015).

[8] A. Aab et al. [Pierre Auger Collaboration], JCAP 1704, 038 (2017).

[9] A. Aab et al. [Pierre Auger Collaboration], Phys. Lett. B 762, 288 (2016).

[10] F. Halzen and H. C. Liu, Phys. Rev. Lett. 48, 771 (1982).

[11] A. Aab et al. [Pierre Auger Collaboration], Phys. Rev. D 91, 032003 (2015); 91, 059901 (2015).

[12] C. Baus, T. Pierog and R. Ulrich, To be published (2016).

[13] T. Pierog et al., Phys. Rev. C 92,034906 (2015).

[14] S. Ostapchenko, Phys. Rev. D 83, 014018 (2011).

[15] D. Heck et al., Report FZKA 6019 (1998).

[16] T. Pierog et al., Proc. 34th ICRC (2015). 